\title{
PSYCHO library for Pure Data
}

\author{
Alexandre Torres Porres \\ 1 \\ EL Locus Solus, independent center for research and computer music \\ Rua Doutor Eduardo de Souza Aranha 122 ap 181 045.43.902 São Paulo, SP
}

el.locus.solus@gmail.com

\begin{abstract}
This paper describes the PSYCHO library for the Pure Data programming language. This library provides novel functions for Pure Data and is a collection of compiled objects, abstractions and patches that include psychoacoustic models and conversions. Most notably, it provides models related to Sensory Dissonance, such as Sharpness, Roughness, Tonalness and Pitch Commonality.

This library is an evolution and revision of earlier research work developed during a masters and $\mathrm{PhD}$ program. The previous developments had not been made easily available as a single and well documented library. Moreover, the work went through a major overhaul, got rid of the dependance of Pd Extended (now an abandoned and unsupported software) and provides new features.

This paper describes the evolution of the early work into the PSYCHO library and presents its main objects, functions and contributions.
\end{abstract}

\section{Early work}

The work presented on this paper started with an older research on the implementation of psychoacoustic models for measuring Sensory Dissonance. The starting point was the implementation of a roughness model during a masters research [1], motivated by its adoption in measuring the dissonance of musical intervals taking into account the spectrum (as developed by Sethares [2]). This was first implemented as a Pure Data patch [3] more precisely, as a Pd Extended patch, as it needed to run in $\mathrm{Pd}$ Extended and relied on some of its built in externals.

During a following $\mathrm{PhD}$ research, in 2009, a compiled external for both Pure Data and MAX was first developed instead of just a patch [4]. A compiled external provides much more CPU efficiency, which is highly needed for a live electronics computer music system such as Pure Data, where you can analyze sounds in realtime for a live performance.

This single external that included only one roughness estimation class evolved into a library as a single binary pack by the end of the $\mathrm{PhD}$ research, but for Pd only [5] (no Max externals have been provided ever since, and the old one is not available anymore). This library contained other classes because the model got more complex and included more features that could also be instantiated as separate objects — such as the conversion from linear amplitude to phons.

In a similar fashion, a library of compiled objects as a single binary pack was developed to include classes related to the Pitch Commonality model as described by Parncutt \& Strasburger [6], including the Tonalness model - these had also been first implemented as Pd Extended patches.

This library, unlike the roughness library, had a modular structure, where the Pitch Commonality model needed to be constructed with a few objects from the library, but the same objects could also be used to derive a Tonalness model, for instance.

Besides these and other compiled objects, a few patches for Pd Extended were also developed during the $\mathrm{PhD}$ research (some of which relying on the externals developed by the author). This body of work has been discussed in previous papers, but as a toolbox [7] and not as the "PSYCHO library". This early work was then available for Pd Extended and only binaries for macOS were provided.

It is important to note that the outcome of the $\mathrm{PhD}$ research was not only the development of computer music tools. It also included an important creative front as a big priority, which applied the provided tools in artistic works and discussed them. Moreover, the thesis provides an extensive and critical review of the psychoacoustic theory, including a psychoacoustic study to better investigate the modeling of psychoacoustic dissonance. Above all, a special attention was given to the review of Roughness modeling theory.

Therefore, the development and implementation of computer music tools during the masters and $\mathrm{PhD}$ was just one of the main pillars of the research. An original contribution was made with the implementation of an original roughness external, plus the first implementations of Pitch Commonality and other objects for Pure Data. Nonetheless, as usual in software development, further developments and enhancements were planned for the future.

\section{Blowing the dust off and revision of the work}

The author lacked technical skills to work independently in coding compiled objects, so a collaboration with others had been necessary for the first developments. Recently, the author has acquired self sufficiency and has worked in the development of other Pure Data libraries ${ }^{1}$. Hence, relying on collaborators is not an obstacle anymore.

1 See $<\underline{\text { https://github.com/porres/pd-else }>\quad \text { and }}$ $<$ https://github.com/porres/pd-cyclone $>$. 
In the meantime, the project was left in the dust, and an issue imposed by the passage of time was the fact that Pd Extended got abandoned and unsupported (and every example patch and abstraction developed relied on it). This isn't that impeditive as one can now easily install libraries from Pd Extended in the main distribution of Pure Data (a.k.a Pd Vanilla). Nonetheless, the most elegant and easiest solution is to offer an independent $\mathrm{Pd}$ library and a body of work that doesn't rely on third party external libraries to run.

This basically required a complete rewrite from scratch to get rid of all externals dependencies and stick to Pd Vanilla (making use of its new features and objects). Besides the example patches, most of the models and compiled objects had also been implemented as Pd Extended patches. As a result, these are now also implemented as pure Pd vanilla patches.

As a more technically fluent programmer, all of the code got also rewritten and revised. In this process, the overall design of the objects went through major surgery as well. As the result, all objects are now available as a separate/single compiled binary, instead of a binary pack that includes many objects as before. This design choice is considered best as it's just more common for Pd libraries to come as separate binaries these days.

The code of each object is also separate now. Hence, the modular structure of the Pitch Commonality model was abandoned and now there's just a single object to measure Tonalness, for example. This major overhaul makes the early work completely obsolete, and is now completely unavailable and lost to oblivion. Besides that, there's also the inclusion of new objects and developments.

\section{The PSYCHO Library for Pd}

This section of the paper describes the main features of the PSYCHO library. At the time of this writing, there's an initial 1.0 release that inaugurates this new incarnation phase of the research. It has been tested and runs on Pd Vanilla 0.50 or later. It is available on $\mathrm{GitHub}^{2}$ and you can also install it via Pd's external manager (a.k.a. 'deken', under Help => Find Externals), just search for "psycho".

\subsection{Roughness}

Roughness is the main dimension of Sensory Dissonance since Helmholtz [8]. The main reference for the provided Roughness model is the work by Clarence Barlow [9], but a revision was proposed to include Vassilakis' amplitude fluctuation degree [10]. The object also offers the complete model by Vassilakis and its main reference (the model of Sethares [2]). You can also tweak the parameters independently to investigate the difference between these parameters and models. The [roughness] object takes as an input a list of frequencies and amplitudes to estimate the roughness.

One of its applications is to draw roughness curves to derive musical scales according to a spectrum. The

$2<\underline{\text { https://github.com/porres/pd-psycho }>}$. [roughcurve] object is an abstraction that uses the [roughness] external to draw dissonance curves (with roughness estimation in the vertical axis and interval in cents on the horizontal axis). It also spits a list of intervals that include an alternation of minimum and maximum points of the curve, which can be used as a musical scale according to the input spectrum.

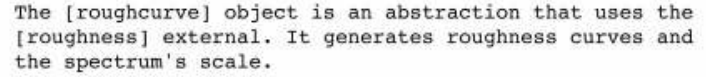
the spectrum's scale.
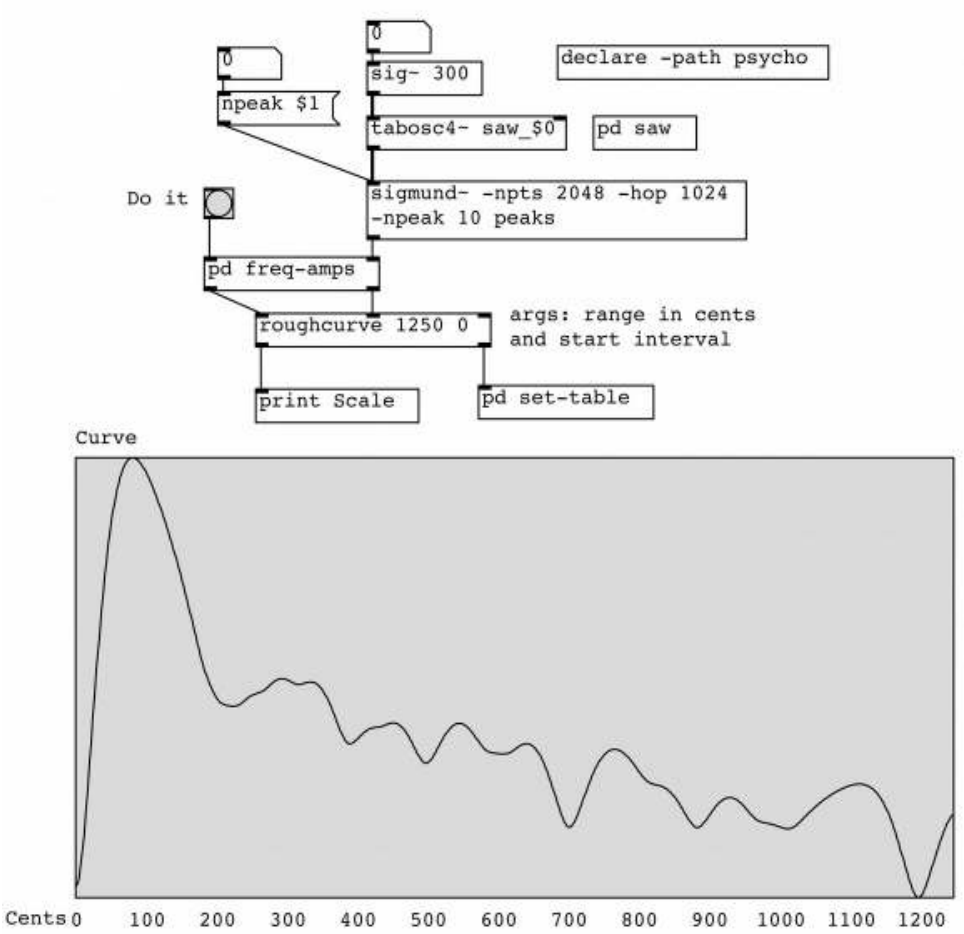

Figure 1: A roughness curve generated with the [roughness] external

\subsection{Indigestibility \& Harmonicity}

These two concepts were developed by Clarence Barlow [11]. The indigestibility of an integer is a measure of how a number may be psychologically 'digestible' according to its prime factors.

Clarence Barlow's harmonicity measure for an interval $p / q$ is defined as the reciprocal of the sum of the indigestibilities of $p$ and $q$, with its sign indicating the interval's polarity. A negative polarity means that the interval is weighted towards its upper tone and a positive number towards its lower tone. As an example, a perfect fifth has its 'weight' on the lower tone, while a perfect fourth has its weight on the highest tone. The absolute value of a harmonicity is called harmonic intensity.

\subsection{Pitch Model objects}

The main reference for the objects in this category is the Pitch Model by Parncutt \& Strasburger [6] which, on its own, is based on the theoretical work of Ernst Terhardt [12] in Pitch \& Consonance, specially Pure/Complex tone Sensation and Sonorousness (which 
is the same as Tonalness). The main outputs of this Pitch Model are: Tonalness, Pitch Commonality and Pitch Distance.

Tonalness is a measure of Pitch clarity. The Pitch Commonality is a measure of the degree in which two sonorities evoke common pitches. The Pitch Distance is a similar concept that considers the probability of noticing a pitch from one sonority in another, but it's more pertinent in the melodic context.

Just like in the Roughness model, the input is a spectrum defined by lists of frequencies and amplitudes. The frequencies list needs to first be converted to Pitch Category (which is basically a logarithmic MIDI scale). On the other side, the amplitude list needs to first be converted to Pure Tone Audibilities (also defined as the Spectral Pitch Weight), which is a perceptual amplitude measure that takes into account a masking level model.

With this new input of frequencies in Pitch Category and amplitudes in Pure Tone Audibilities, we can recognize a harmonic pattern. The Complex Tone Audibility $\operatorname{Ac}(\mathrm{P})$ (or Virtual Pitch Weight) is a degree to which a Harmonic Series (or part of it) is present in the spectrum. For that, a template of 10 harmonics is used, each with a different weight. When there's a match, the Complex Tone Audibility value is registered according to the Pure Tone Audibilities. If a Pure and Complex Tones Audibilities have the same Pitch Category, the greater Audibility value is registered as the Virtual Pitch Weight.
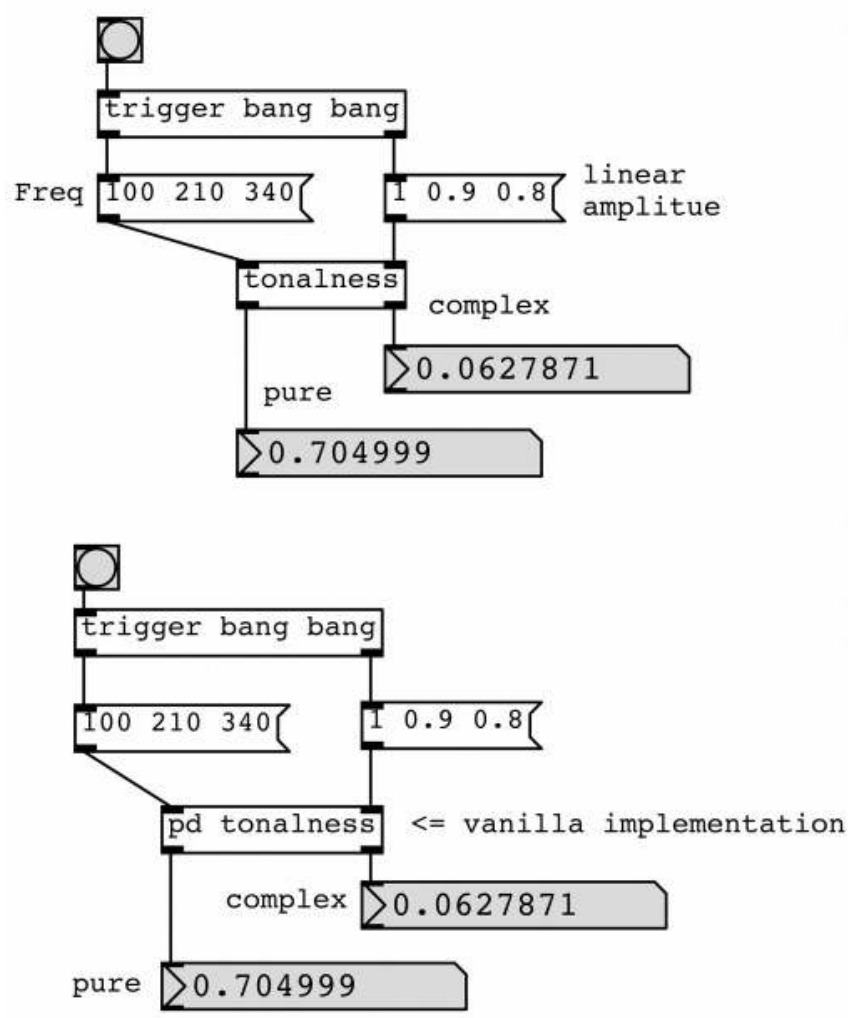

Figure 2: Pure \& Complex Tonalness measure with the [tonalness] external and a Pd Vanilla patch
The Pure Tonalness estimation is a normalized quadratic sum of Pure Tone Audibilities Ap(P), and the Complex Tonalness is derived from the maximum Complex Tone Audibility Ac(P). The [tonalness] object outputs both measures. You can set the number of pitch categories. The default is 12 pitches per octave and 10 octaves (hence, an array of 120 values).

Pitch Multiplicity is the number of tones consciously perceived in a sonority. It is calculated from Pitch Audibility A(P), which is given by the maximum value of Pure and Complex Tone Audibilities for each Pitch Category. The Tone Salience is the probability of consciously perceiving a pitch and depends on both Pitch Audibility and Pitch Multiplicity. The [salience] object outputs both the multiplicity value and the Pitch Salience profile, which is the list of Pitch Salience for each Pitch categories (120 by default, like the [tonalness] object).

Successive Pitch Relationships are given by Pitch Commonality and Pitch Distance, both dependent on the Tone Salience output. The Pitch Commonality measure is a Pearson correlation coefficient between two Tone Salience profiles and is given the [commonality] object. The relationship increases according to common Tone Saliences and is equal to 1 in the case of equal spectra and -1 in the case of supposedly complementary ones.
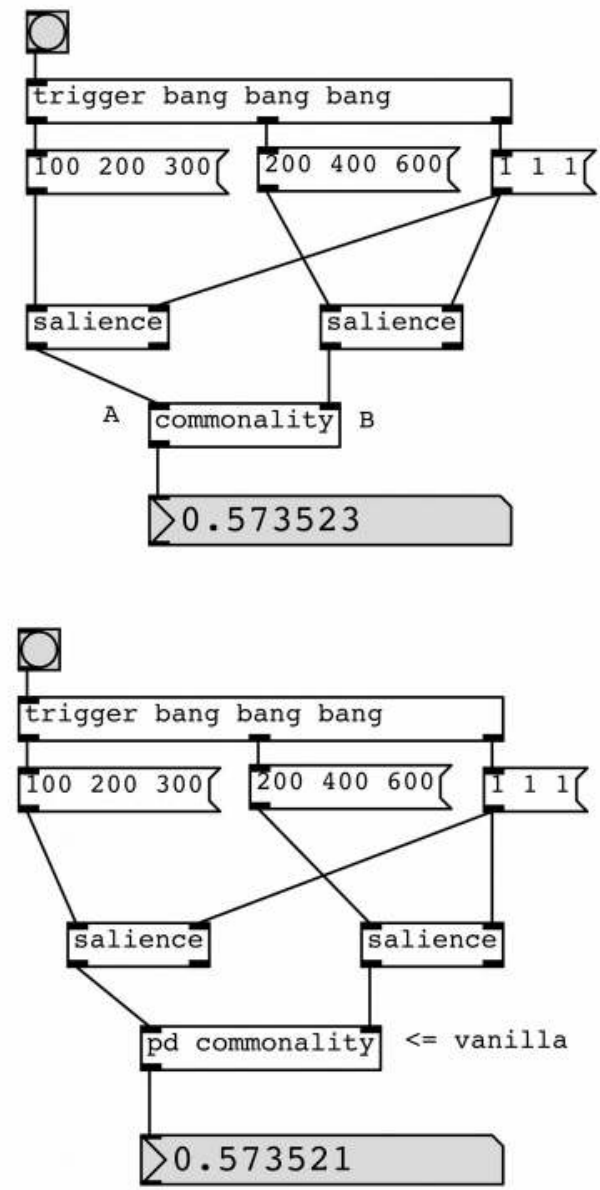

Figure 3: Pitch Commonality measure from Pitch Salience Profiles with the [commonality] external and a Pd Vanilla patch 
The Pitch Distance is given by the [distance] object and considers the probability of noticing a pitch from one sonority in another. It takes into account all possible intervals between perceived pitches in two sonorities. The distance is zero for identical sonorities, and exceeds zero otherwise. In the case of Pure Tones, the Pitch Distance is equal to the interval between them in semitones.

\subsection{Other objects}

This section highlights other relevant objects not presented in the previous sections. Regarding dissonance, the library also contains a [sharpness ] object, which is also part of the Sensory Dissonance Model (and defined as the perceptual equivalent to the spectral centroid). A [centroid ] object is also available as it relates to the measure of sharpness. Also related is the [barks ] object that measures spectral energy per critical band/bark.

Most of the other objects convert frequencies and amplitudes to psychoacoustic scales. For example, for frequencies, we have [hz2bark] and [hz2mel]. For amplitudes, there are objects that convert to Phons, which can be used to plot Equal Loudness Curves.

\subsection{Future objects and additions for further releases}

Not everything that was developed from the $\mathrm{PhD}$ has been fully revised and ported yet, but this is planned for future releases. Currently in the workings, there are a few patches that processes live input. The most important one is a spectral process that alters the spectra relationships of partials to match a given scale. This is known as the spectral mapping technique, also provided in the spectral tools set of objects for Max/MSP [13].

\section{Final Considerations and Further work}

This paper presented the PSYCHO library for Pure Data, which focuses on psychoacoustic descriptors of Dissonance and is the first library to do so for Pd. It also includes psychoacoustic models never implemented for real time computer music systems, such as the Pitch Commonality model.

This library revisits and restores an earlier research work that had been left virtually abandoned. A drastic makeover provides several advantages such as a friendlier design, a proper documentation, the independence from third party externals and a revision of the code.

The library is also expanding to include audio descriptors as the [centroid $\sim$ ] and [barks ] object. Other objects that are actually low level descriptors are also being considered to be part of the library. For example, raw cepstrum, mel-frequency cepstrum and Barkfrequency cepstrum, which have already been used in Pd for measuring timbral characteristics [14].

\section{References}

[1] Porres, A.T. Processos de composição microtonal por meio do modelo de dissonância sensorial, Master dissertation, Unicamp, 2007.

[2] W. Sethares, Tuning, Timbre, Spectrum, Scale (2nd ed.), Springer-Verlag, 2004.

[3] Porres, A.T. "A Roughness Model for An Adaptive Tuning Patch Controlled by Antennas" PdCon07 (2nd International Pure Data Convention) Montreal, 2007.

[4] Porres, A. T. and Pires, A.S. (2009). "Um External de Aspereza para Puredata \& MAX/MSP," in Proceedings of the 12th Brazilian Symposium on Computer Music 2009 (SBCM 2009). Recife, PE: Braz

[5] Porres, A. T. Modelos psicoacústicos de dissonância para eletrônica ao vivo, PhD thesis, USP, 2012.

[6] R. Parncutt, H. Strasburger, "Applying psychoacoustics in composition: "Harmonic" progressions of "non-harmonic" sonorities", Perspectives of New Music, 1994, 32 (2), pp. 1-42.

[7] Porres, A.T. "Dissonance Model Toolbox in Pure Data" Proceedings of the 4th Pure Data International Convention (PdCon11), Berlin, Germany, 2011.

[8] H. Helmholtz, On the Sensations of Tone as a Psychological basis for the Theory of Music (2nd ed.), Dover Publications, 1954.

[9] C. Barlow, "Von der Musiquantenlehre" Feedback Paper 34, 2008. Feedback Studio Verlag.

[10] P.N. Vassilakis. "Perceptual and Physical Properties of Amplitude Fluctuation and their Musical Significance.” Ph.D. Thesis, 2001, UCLA.

[11] Barlow, Clarence \& Lohner, Henning. (1988). Two Essays on Theory. Computer Music Journal. 11. 44.

[12] E. Terhardt, "The concept of musical consonance: A link between music and psychoacoustics", Music Perception, 1984, Vol.1, pp. 276-295.

[13] W. A. Sethares, A. J. Milne, S. Tiedje, "Spectral Tools for Dynamic Tonality and Audio Morphing" Computer Music Journal, 2009, Volume 33, Number 2, Summer 2009, pp. 71-84.

[14] Brent, William. Perceptually Based Pitch Scales in Cepstral Techniques for Percussive Timbre Identification: a comparison of raw cepstrum, mel-frequency cepstrum, and Bark-frequency cepstrum. From the proceedings of the ICMC in Montreal, 2009. 